\title{
Computer Circuit Lab Experiments Using Limited Lab Resources
}

\author{
Christopher R. Carroll \\ University of Minnesota Duluth
}

\begin{abstract}
Lab experiments in which students design and test functional parts of digital computers are useful endeavors that demonstrate how computer functions can be implemented and that reduce the mystery that surrounds computer circuits in students' minds. However, generating lab assignments that both involve significant processing functions and require manageable amounts of hardware is a daunting task for course instructors. Many educators have turned to simulation of digital circuits that implement computer functions, a decision that removes the student from the actual hands-on contact with circuits and components that are involved in computer design. There is no better way to gain a gut feeling for how a circuit functions than to build it and make it work, including understanding the curious circuit behaviors that can result from misplaced wires or faulty components. Simulation separates the student from the operation of the circuit, and can increase the student's perception that the circuit is "too complicated" or "too mysterious" to implement on a breadboard.
\end{abstract}

Often the most cumbersome part of a computer circuit implementation is providing input and output interfacing. The author developed a simple microcontroller-based instrument that implements a 12-key keypad input and a 16-character hexadecimal display for use in interfacing digital circuits, which was reported at an earlier ASEE conference ${ }^{1}$. This paper reveals several lab experiments that have been performed by students to investigate digital computer functions, including arithmetic circuits such as multipliers and dividers and data structure implementations such as queues (FIFOs) and stacks (LIFOs). These lab experiments all have been completed by students using just a handful of standard logic chips plus the author's input/output instrument mentioned above. By breadboarding these circuits, rather than simulating them, students obtain a fuller understanding of how the circuits work and gain confidence in the design of computer systems.

\section{Carroll's Gadget}

The instrument that makes developing manageable computer circuit lab exercises easy is known as Carroll's Gadget. Its design was reported in an earlier ASEE conference paper $^{1}$. This instrument, designed and built by the author, is housed in an old telephone handset and consists of a microcontroller-based circuit that uses the 12-key telephone keypad as an input devices and that generates a display of up to sixteen hexadecimal characters on a standard oscilloscope screen for use as an output device. The instrument 
is shown in Figure 1, and the initial display produced upon powering the instrument is shown in Figure 2. Interface to these input and output devices is intentionally simple so that they can be incorporated easily into student designs for various types of computer circuits. By removing the need to worry about input/output, students can concentrate on the actual data processing or storage circuits they are designing.

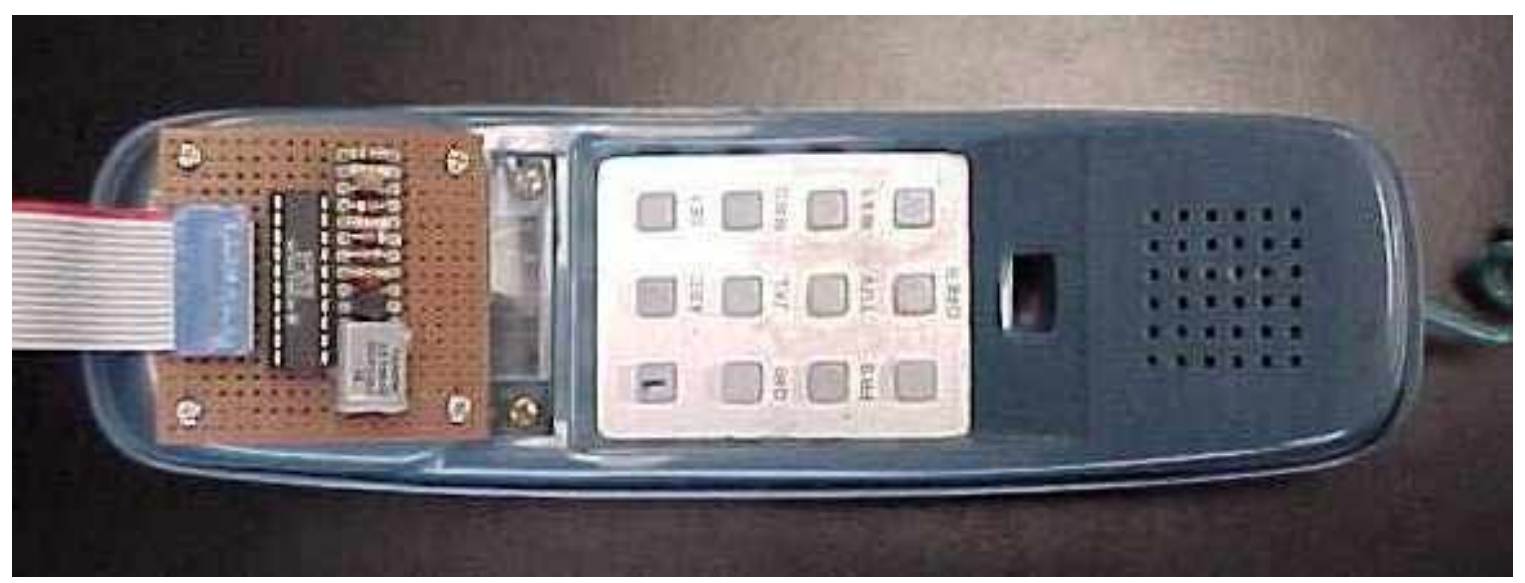

Figure 1. Carroll's Gadget

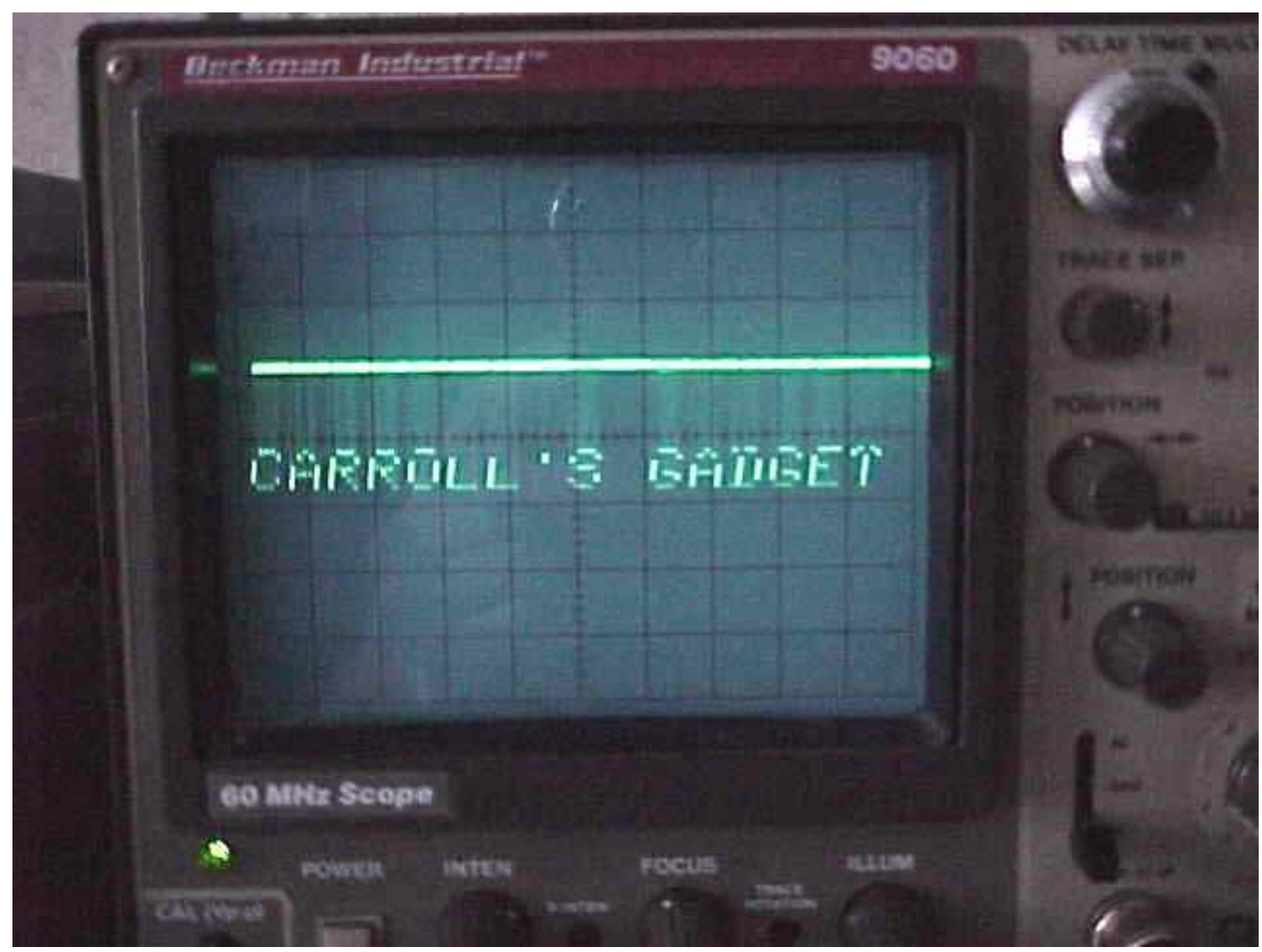

Figure 2. Power-on display produced by Carroll's Gadget 
The twelve-key keypad input device supplies a four-bit key value to the user's circuit, and a one-bit "valid" variable to indicate a key press. The sixteen-digit display accepts a four-bit value from the user's circuit each clock cycle to indicate what hexadecimal character to show, a one-bit signal that says whether or not to actually show a digit that clock cycle, and another one-bit signal that says whether to reposition the invisible "cursor" to the left side of the display or to allow the "cursor" to advance to the next character position to the right. This simple set of signals allows students to interface their computer circuit designs to the Gadget's input/output devices without confusion.

Carroll's Gadget is a very simple instrument electrically. Figure 3 shows a close up of the complete circuit, as implemented in the telephone handset housing. It is a microcontroller-based design, and requires only 5-volt power from the user's circuit to operate. A single ribbon cable connects the Gadget to the user's circuit, and the phone's coiled cord conveys the display information to the oscilloscope.

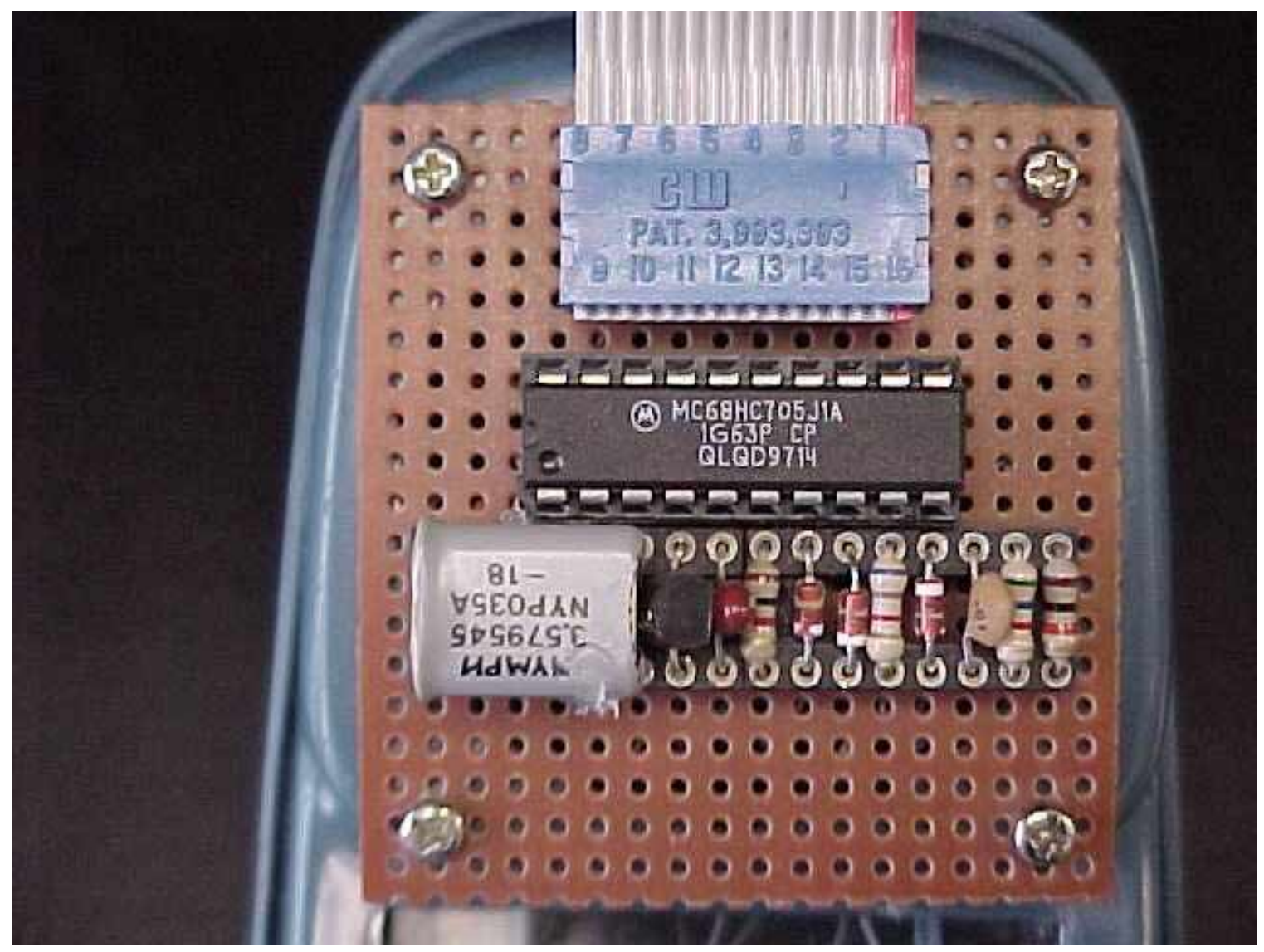

Figure 3. Close-up of Carroll's Gadget electronics

Carroll's Gadget can be used to interface a variety of digital computer functional circuit blocks as student lab experiments. Following some initial lab assignments to acquaint students with the Gadget's operation, lab exercises fall into two groups, data manipulation and data structure implementation. Figure 4 shows the Gadget's oscilloscope screen displaying each of the sixteen possible hexadecimal characters, used as output for student design experiments. 


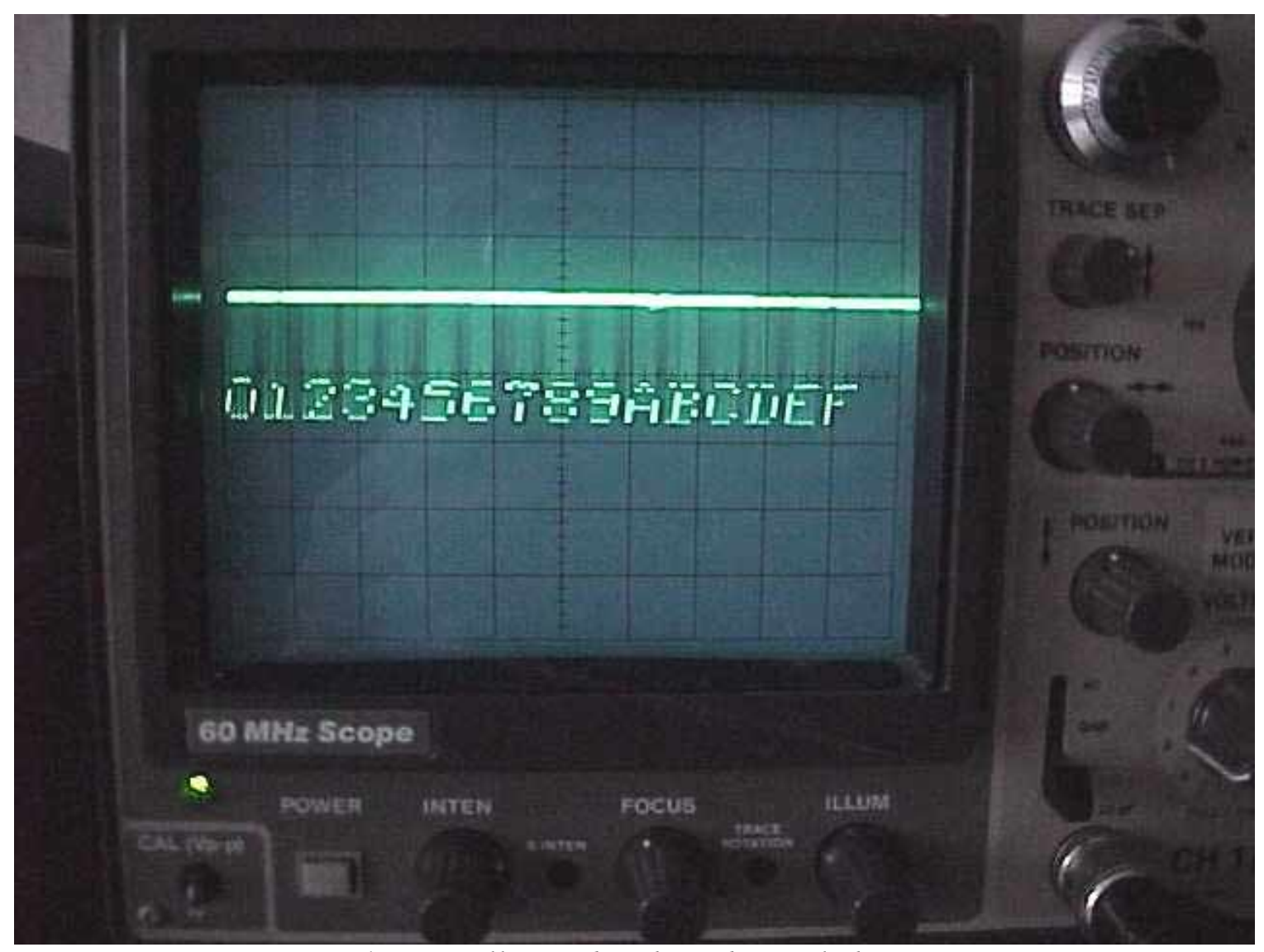

Figure 4. Carroll's Gadget hexadecimal character set

\section{Data Manipulation Circuits}

Data manipulation includes calculation or modification of information. Arithmetic circuits implementing adders, subtracters, multipliers, and dividers are all possible, as are circuits that modify data stored in standard memory chips in various ways.

A typical arithmetic lab assignment is a shift-and-add multiplier. Students design a circuit that captures two single-digit hexadecimal numbers entered from the Gadget's keypad, multiplies them, and displays the result as a two-digit hexadecimal number on the Gadget's oscilloscope display. This four-bit by four-bit multiplier can be completed using only ten standard TTL chips plus the Gadget, certainly a manageable circuit for students to build, and much easier to understand and debug than if students had to design and build their own input/output circuits in addition to the multiplier itself. This assignment requires students to employ in their designs a variety of Medium Scale Integration (MSI) components, such as adders, counters, and shift registers.

Another assignment requires students to modify information stored in a standard $1 \mathrm{~K} x 4$ RAM chip. For example, sixteen locations in the RAM are continuously displayed on the Gadget oscilloscope display as sixteen hexadecimal numbers. A single keystroke on the Gadget keypad identifies one of those values in the RAM by specifying its address, and the data at that address is read, incremented, and written back to the same location. This 
exercise requires students to understand the timing involved in interacting with the RAM data during both the read and write processes. It provides an opportunity to introduce multi-phase clocking, which is also supported by the Gadget instrument.

\section{Data Structure Implementations}

Another category of computer circuits that forms the basis for lab experiments using Carroll's Gadget involves hardware implementation of various data structures. Typical examples are a stack, or Last in First Out (LIFO) data structure, and a queue, or First In First Out (FIFO) data structure.

The LIFO lab uses a single twelve-bit shift register to store four three-bit numbers continuously circulating. The four numbers are displayed on the Gadget oscilloscope display as four digits. As non-zero numbers are entered from the keypad, each new number is "pushed" onto the stack, appearing on the left side of the display and bumping the digit that was on the right off the end of the stack. Pushing a zero on the keypad "pops" the stack, removing the digit on the left of the display and supplying a zero in the vacated position on the right end. This lab provides an opportunity to introduce pseudosynchronous clocking, or multiple clocks derived from the same master clock, since most of the circuit is manipulating information in the twelve-bit shift register at the digit level, i.e. every three bit times, rather than at the rate at which the individual bits are shifting.

The FIFO lab uses a 4 x 4 dual-port RAM chip, the 74LS670, to store up to four hexadecimal digits entered from the Gadget keypad. The * key is used to extract the oldest entry from the FIFO. The contents of the FIFO are continuously displayed on the Gadget display, which shows nothing when the FIFO is empty, and then fills to a maximum of four digits when the FIFO is full. This lab exercise gives students another chance to manipulate data in a RAM using multi-phase clocking, and demonstrates the dual-port feature that makes reading and writing this RAM completely independent of each other.

\section{Summary}

Carroll's Gadget has proven to be a remarkably versatile instrument. By providing useful input and output devices in the Gadget, students are able to design and build complete pieces of digital computer circuits implementing data processing or data structure circuits without the extra complexity involved in getting data in or out of their designs.. The instrument has been used for several semesters in the "Computer Circuits" laboratory of the Electrical and Computer Engineering department at the University of Minnesota Duluth, and has been dependable and reliable, despite rugged use by many students. The Gadget is inexpensive and uncomplicated, making it a very non-intimidating test instrument in the lab. Carroll's Gadget demonstrates that test equipment need not be expensive or intimidating to be effective in an educational lab environment. 


\section{Bibliography}

1. Carroll, C. R., "Portable Input/Output Instrument for Interfacing Student Digital System Designs," 1998 ASEE Annual Conference Proceedings, Seattle, WA (1998).

\section{Biography}

\section{CHRISTOPHER R. CARROLL}

Christopher R. Carroll received a Bachelor of Engineering Science from Georgia Tech, and M.S. and Ph.D. degrees from Caltech. After teaching in Electrical Engineering at Duke University, he is now Associate Professor and Assistant Head of Electrical and Computer Engineering at the University of Minnesota Duluth. His interests include special-purpose digital systems, VLSI, and microprocessor applications. 